\title{
A singular case of intravesical bleeding angiomyolipoma in a bladder diverticulum
}

\author{
Michael E. Chua, MD; Maria Anna Vanessa A. Geron, MD \\ Institute of Urology, St. Luke's Medical Center, Philippines
}

Cite as: Can Urol Assoc J 2013;7;E125-E129. http://dx.doi.org/10.5489/cuaj.254

\section{Abstract}

Neoplasms arising from intravesical diverticula are rare and considered by urologists as an important surgical challenge. A hamartomatous lesion noted in a bladder diverticulum has never been reported. To our knowledge, we report the first angiomyolipoma, a subtype of mesenchymal hamartoma uncommonly located extrarenally, seen in a bladder dome diverticulum. We discuss the dilemma on the management of such case, related literature and probable etiology.

\section{Introduction}

Bladder neoplasm arising within a diverticulum is uncommon, with an overall reported incidence varying from $0.8 \%$ to $10 \%$, all of which are histologically malignant in origin. ${ }^{1}$ Currently, there is no literature of a hamartoma arising from a bladder diverticulum. Angiomyolipoma, classified as mesenchymal hamartoma, is considered rare in extrarenal locations, particularly in the bladder. ${ }^{2,3}$ To our knowledge, this is the first case of angiomyolipoma arising from an intravesical diverticulum. This case represents a surgical challenge that may serve as a reference for the management of similar situations.

\section{Case report}

A 58-year-old Asian-American male was referred to the urology service of a tertiary hospital with a history of painless total gross hematuria. He experienced continuous gross hematuria for 1 week, initially light red in colour, later progressing to dark red urine. This was associated with

urinary retention secondary to clot formation. No pain or any other systemic symptoms were noted. The patient is hypertensive, non-diabetic and a non-smoker. He worked as a hospital staff worker with no history of any industrial chemical exposure. The physical examination, including digital rectal examination of the prostate, was unremarkable. Urine red blood cell (RBC) morphology revealed 100 to 120 isomorphic RBC per high power field, and urine cytology was negative. Decreased hemoglobin of $12.2 \mathrm{~g} /$ $\mathrm{dL}$ (range: 13-17 g/dL) was also noted. Other blood works, including serum creatinine, serum prostate-specific antigen and bleeding parameters were unremarkable. Abdominopelvic computed tomography with triple contrast study (oral, intravenous and intravesical) showed a well-distended urinary bladder with a focal outpouching noted at the dome of the bladder with hypodensities within (Fig. 1, coronal, Fig. 2, sagittal). The patient then underwent rigid cystourethroscopy under spinal anesthesia, with findings of smooth uretha, non-obstructing prostate lobes, mildly trabeculated bladder wall, normal bilateral ureteral orifice and no active bleeding. As the bladder was filled, there was note of a diverticulum at the bladder dome (Fig. 3a). Its base was broad with a friable yellowish mass emanating from within the diverticulum (Fig. 3b). Initially, transurethral resection of bladder tumour (TURBT) was attempted, but technical difficulties were encountered due to the location of the diverticulum. Also, considering its thin-walled nature, risk of diverticular perforation, and subsequent peritoneal tumour spillage is high. Therefore, cup biopsy was done on the base and the superficial area of the mass. The histopathology result of the specimen revealed fibroadipose tissue.

Two days after the biopsy, he had a recurrence of gross hematuria with further decrease in hemoglobin $(9.2 \mathrm{~g} / \mathrm{dL})$. A multidisciplinary meeting was held to discuss the management of the case, with particular attention to the hemor- 


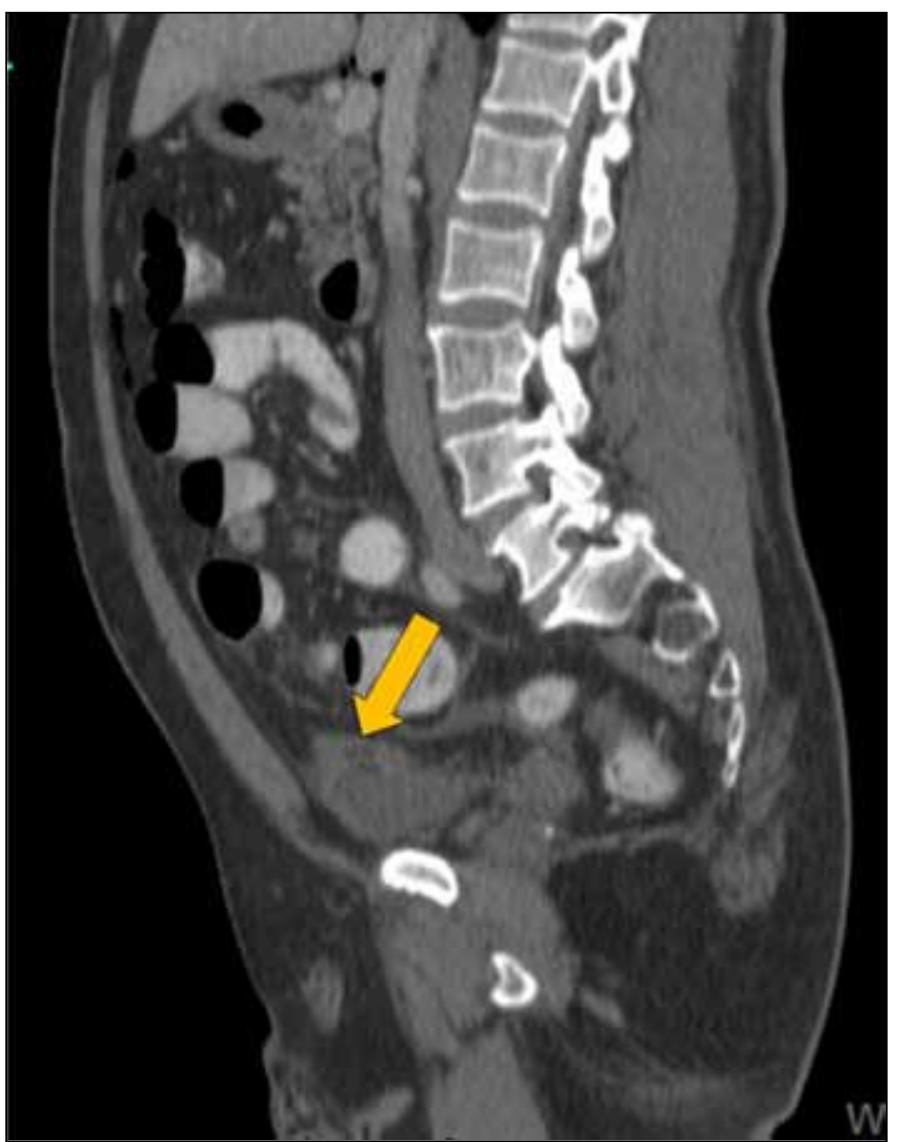

Fig. 1. Abdomino-pelvic computed tomography scan with intravenous and oral contrast (sagittal view): Arrow points on the thinned portion of the bladder dome, with noted intraluminal inhomogenous structure.

rhagic complications. The team was also concerned with the possibility of inadequate specimen obtained from a cup biopsy, yielding a false negative result. Should the tumour be malignant in origin, peritoneal extension must be dealt with accordingly. Hence, the patient was advised to undergo an open partial cystectomy, which was thought to give better exposure involving the area concerned. This was also the surgical approach of choice to evaluate and address any possible peritoneal extension. At surgery, there was note of adhesions between the bladder dome and adjacent colonic segment. Adhesiolysis was done and the bladder was mobilized. Partial cystectomy was done at the bladder dome, encompassing the bladder diverticulum. Grossly, a $3.2 \times 3.1 \times 1.8-\mathrm{cm}$ well-delineated, firm, exophytic mass with cream tan, granular surface was noted projecting from the portion of smooth glistening lining of the bladder diverticulum (Fig. 4). Histologically, the mass was composed of mature adipose tissue admixed with fibrous tissue, blood vessels and smooth muscle fibres (Fig. 5). The cytomorphological and histochemical study concluded the diagnosis of an angiomyolipoma in a bladder diverticulum. He was discharged on postoperative day 3 without any complications. On the sixth month following the procedure, a CT scan of

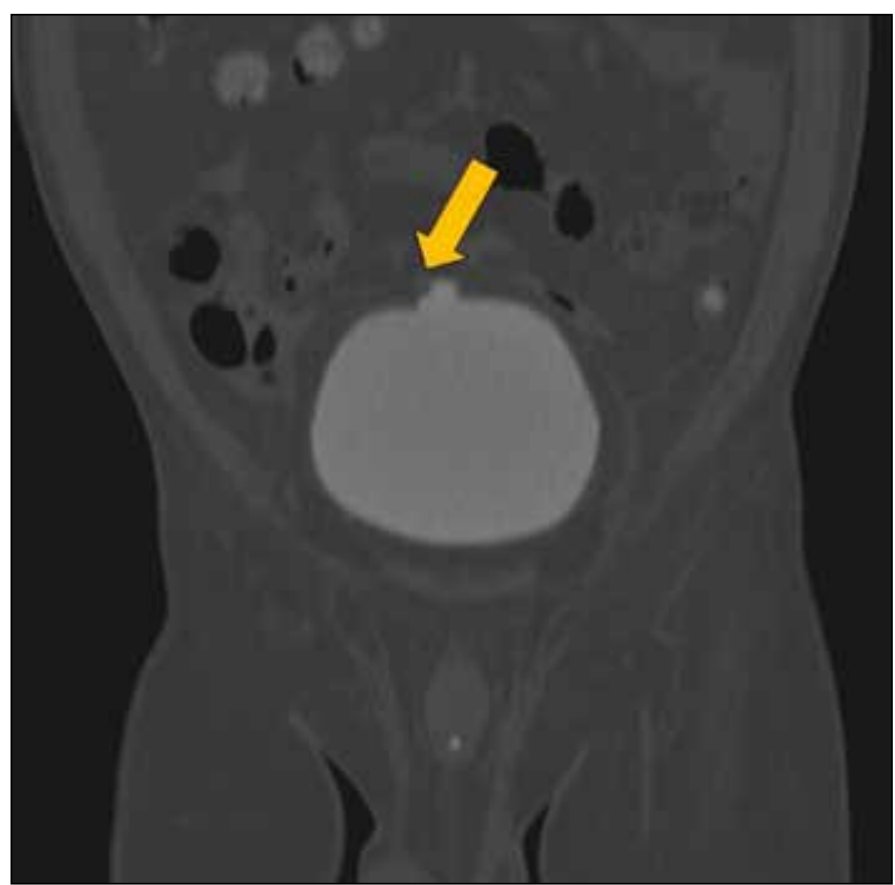

Fig. 2. Abdomino-pelvic computed tomography scan with intravesical filled contrast (coronal view): Arrow points at the focal outpouching with inhomogenous structure on the bladder dome.

the whole abdomen was unremarkable and the patient had no recurrence of gross hematuria.

\section{Discussion}

Bladder diverticula are considered pseudodiverticula formed by mucosal herniations from areas of acquired or congenital weakness in the muscular bladder wall. These occur secondary to increased intravesical pressure due to bladder outlet obstruction. Occasionally, bladder diverticulum may result from congenital disarrangement of muscle fibers, mostly located in the ureterovesical junction. This is commonly referred to as "Hutch" diverticula. ${ }^{1}$ In our case, the latter is the most likely etiology.

Painless hematuria in a 50-year-old male, unless proven otherwise, should indicate a malignant neoplasm of the bladder. Our patient presented with painless gross hematuria and urinary retention, which are usually the presenting symptoms of the neoplastic changes in bladder urothelium. ${ }^{4,5}$ The incidence of neoplastic changes within the bladder diverticulum is estimated at $2 \%$ to $10 \%{ }^{6}{ }^{6}$ There is still no clear pathophysiologic explanation behind these neoplastic changes. Urinary stasis in these areas may induce chronic mucosal irritation and prolong exposure to urinary carcinogens; hence, the predisposition of the diverticular urothelium to a malignant degeneration. ${ }^{7}$ Due to its association with grave prognosis, a high index of suspicion and prompt diagnosis are needed to treat patients with tumours within bladder diverticula. ${ }^{1}$ 


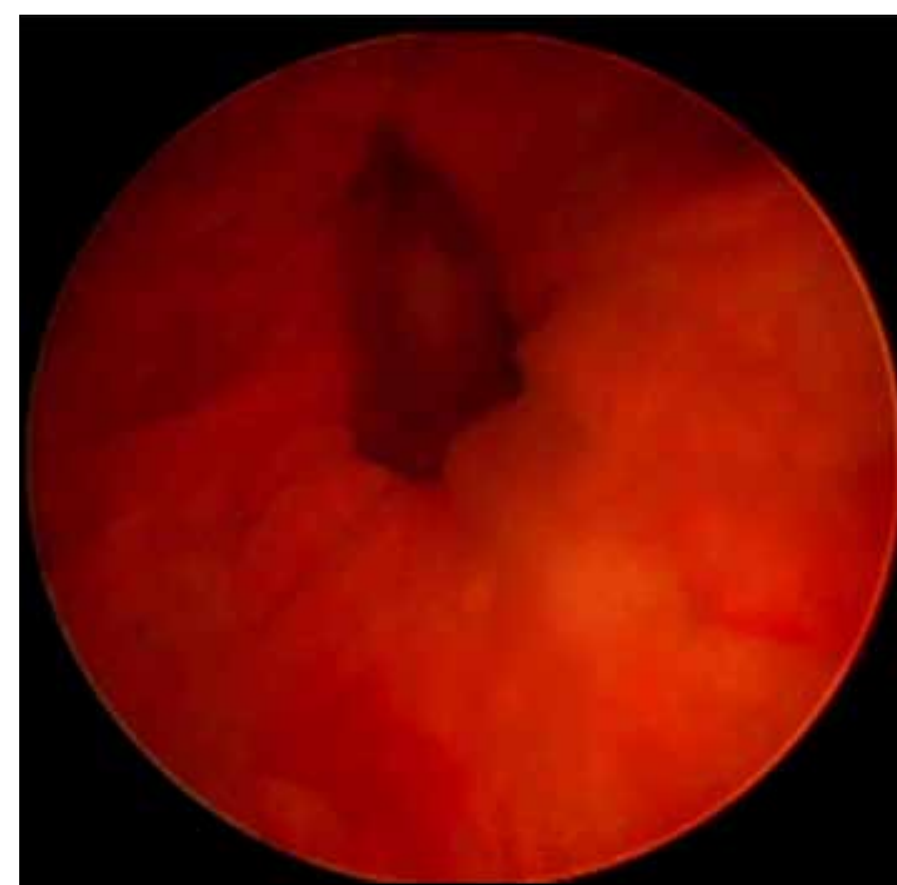

Fig. 3a. Cystoscopic finding of bladder diverticulum opening located at the bladder dome.

A recent pathological review done at Cleveland Clinic, Ohio, USA, revealed that malignant neoplastic changes noted in these bladder diverticular tumors were present in 36 out of 71 cases $(51 \%)$, including both noninvasive and invasive carcinoma. The most common histologic finding is papillary urothelial carcinoma; less common subtypes include small cell carcinoma, squamous cell carcinoma and adenocarcinoma. Benign diverticular lesions usually involve inflammatory histologic changes, such as cystitis cystica and cystitis glandularis, tissue granuloma and cellular atypia. ${ }^{8}$ Negative urine cytology in suspicious cases do not provide a good sensitivity to rule out urothelial cancers; ${ }^{9}$ moreover, both neoplastic and benign diverticula possess fibrovascular stromal tissues, such as fibroadipose tissue, which is usually found on the dense fibrous tissues that define the boundary between perivesical fat from the lamina propia. ${ }^{6}$ Therefore, the above-mentioned pathological tissue changes in intravesical diverticulum were all considered differential diagnoses in our case.

In general, neoplasms arising from intravesical diverticulum present a diagnostic and therapeutic difficulty. ${ }^{4}$ Due to its rarity and lack of systematic clinical studies, there are currently no available guidelines regarding the management of neoplasms arising from intravesical diverticulum. With malignant neoplasms confined to the diverticulum, there is better prognosis than those with extravesical extension. These can also be managed conservatively either by transurethral resection or partial cystectomy with the overall disease-free survival rate comparable to those who underwent

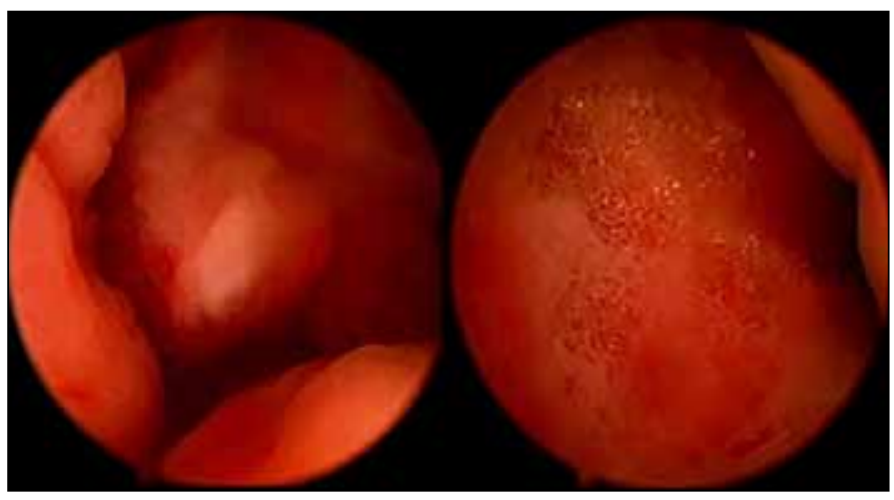

Fig. 3b. Cystoscopic finding of friable yellowish tissue emanating from the diverticulum. On closer view of the mass, fibro-fatty component of the surface was noted.

radical cystectomy. ${ }^{1,6}$ An algorithm for the management of intravesical diverticulum with transitional cell carcinoma was proposed by Raheem and colleague. ${ }^{11}$ In this management algorithm, factors that affect the choice of surgical approach (conservative vs. radical) include anesthesia risk, WHO grade, TNM staging, size of diverticular neck, thickness of diverticular wall, freedom from other intravesical tumours and location of diverticulum relative to ureteric orifices. ${ }^{11}$ In our case, conservative management by TURBT was initially considered; however, this was deferred mainly due to the wall thickness and location of the divericuli. These features suggest higher risk of perforation and peritoneal spillage. The team decided to address the hemorrhagic complications, increased risk of false negative results from cup biopsy, and risk of peritoneal extension, with open partial cystectomy. This surgical approach was considered more conservative with less morbidity as compared to radical cystectomy.

With the final histopathologic findings of angiomyolipoma in a bladder diverticulum, this was, to our knowledge, the first report regarding a hamartoma arising from an intravesical diverticula. Hamartomas are malformations that present as a mass of disorganized tissue indigenous to a particular site. ${ }^{12}$ It also entails an excessive focal overgrowth of normal mature tissues in an organ, but do not reproduce the normal architecture of the surrounding tissue. ${ }^{13}$ Hamartomas can arise from any organ, but those of the urinary bladder are extremely rare. Angiomyolipoma, a kind of mesenchymal hamartoma, is a malformation of tissue elements forming a tumour that is haphazardly arranged. This originates from a single cell with the ability of pluripotential differentiation, demonstrating co-expression of muscle, adipose and vascular components. ${ }^{12}$ The kidney and liver are the most commonly affected organs; currently, there are only three reported cases of angiomyolipoma involvement in the perivesical wall ${ }^{2,3,14}$ and one located in the intravesical floor. ${ }^{15}$

We speculate that both bladder diverticulum in the dome and the component of hamartomatous angiomyolipoma in 


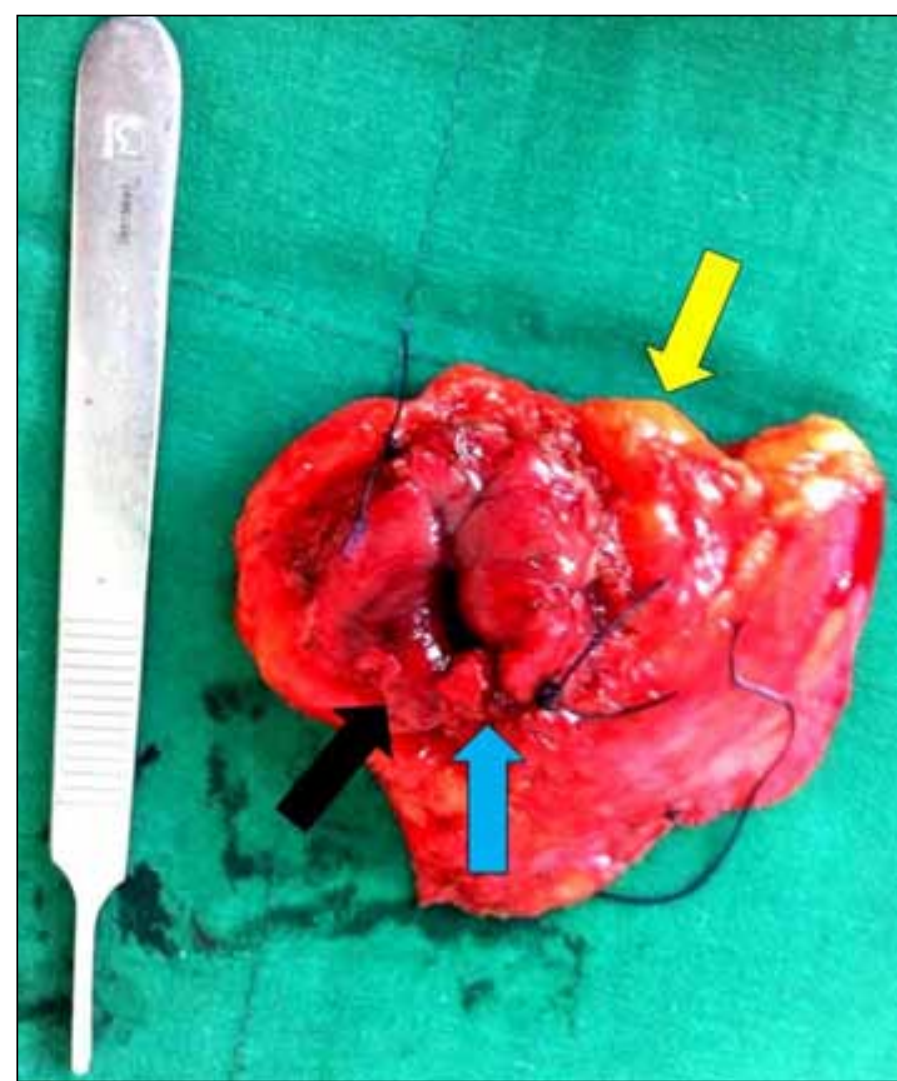

Fig. 4. Gross specimen view from bladder lumen side up: Blue arrow pointing on the yellowish mass projecting from the bladder diverticulum. Black arrow pointing on the intravesical diverticulum. Yellow arrow pointing on the perivesical fat.

our case was a result of embryogenic malformation. In the normal embryologic development of the urogenital system, the cranial part of the urogenital sinus is continuous with the allantois, developing into the bladder and pelvic urethra; the bladder dome, posterior walls and part of lateral walls, normally arise from surrounding urogenital sinus mesenchyme. ${ }^{16}$ In recent investigation of embryonic development of bladder, Shapiro and colleagues described that bladder morphogenesis is regulated by complex epithelial-mesenchymal signalling events. ${ }^{17}$ Failure of these signalling events to occur at specified times result in bladder anomalies. Our case supports the proposed notion that the components and appearance of hamartomas and diverticula, when present simultaneously in uncommon locations, is a result of malformation attributed to improper proportion and distribution of fetal mesenchymal elements in embryonic development. ${ }^{3,8}$

\section{Conclusion}

The clinical manifestations and radiologic appearance of hamartomas arising from the bladder, particularly in an intravesical diverticulum are nonspecific. It is impossible to differentiate it from a malignant neoplastic process on

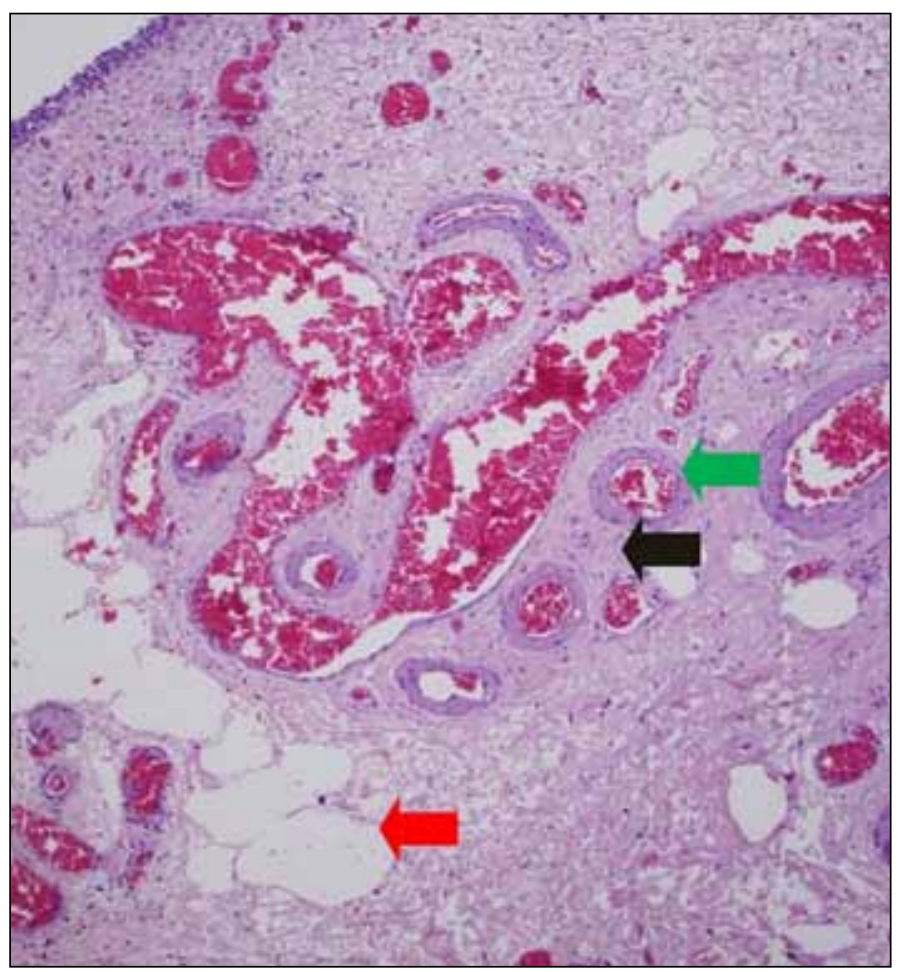

Fig. 5. Histology result of intravesical diverticulum mass with hematoxylin \& eosin stain on high power view (microscopic magnification $=40 \times$ objectives). Green arrow pointing at a blood vessel with intraluminal blood cells. Black arrow pointing at mature smooth muscle cells. Red arrow pointing at mature fat cells

the basis of radiologic results or physical examination; only a complete pathologic study is the definitive diagnostic modality. ${ }^{10}$ Moreover, a conservative management for similar cases may be considered depending on the condition of the diverticulum and extent of the neoplasm; however, with our case, the presentation of recurrent hematuria and associated complications warrant urgent partial cystectomy.

Competing interests: None declared.

This paper has been peer-reviewed.

\section{References}

1. Golijanin D, Yossepowitch 0, Beck SD, et al. Carcinoma in a Bladder Diverticulum: Presentation and Treatment Outcome. J Urol 2003;170:1761-2. http://dx.doi.org/10.1097/01.ju.0000091800.15071.52

2. De Siati M, Visona A, Shah J, et al. Angiomyolipoma of the Bladder Wall. J Urol 2000;163:901-2. http://dx.doi.org/10.1016/S0022-5347(05)67833-0

3. Constantinides $C A$, Giannopoulos A, Stinios J, et al. Angiomyolypoma of the Left Ureterovesical Junction Associated with Ipsilateral Renal Agenesis. J Urol 1995;153:1897-8. http://dx.doi.org/10.1016/ S0022-5347(01)67345-2

4. Cheng CW, Ng MT, Cheung HY, et al. Carcinosarcoma of the bladder diverticulum and a review of the literature. Int. J Urol 2004;11:1136-8. http://dx.doi.org/10.1111/j.1442-2042.2004.00956.x

5. O'Donnell MA, Lilli K, Leopold C. Contemporary Profile of Superficial Bladder Cancer as Revealed from an Open-entry National Muticenter Study. J Urol 2004;171:75A. 
Intravesical bleeding angiomyolipoma in a bladder diverticulum

6. Moinzadeh A, Latini J, Hamawy KJ. Clear Cell Adenocarcinoma of the Urinary Bladder within a Diverticulum. Urology 2003;62:145. http://dx.doi.org/10.1016/S0090-4295(03)00242-5

7. Matta EJ, Kenney AJ, Barré GM, et al. Intradiverticular Bladder Carcinoma. RadioGraphics 2005;2:1397403. http://dx.doi.org/10.1148/rg.255045205

8. Tamas EF, Stephenson AJ, Campbell SC, et al. Histopathologic Features and Clinical Outcomes in 71 Cases of Bladder Diverticula. Arch Pathol Lab Med 2009;133:791-6.

9. Schwalb DM, Herr HW, Fair WR. The Management of Clinically Unconfirmed Positive Urinary Cytology. J Urol 1993; 150:1751.

10. Brancatelli $G$, Midiri $M$, Sparacia $G$, et al. Hamartoma of the urinary bladder: case report and review of the literature. Eur Radiol 1999;9:42-4. http://dx.doi.org/10.1007/s003300050624

11. Raheem OA, Besharatian B, Hickey DP. Surgical Management of Bladder Transitional Cell Carcinoma in a Cesicular Diverticulum: Case Report. Can Urol Assoc J 2011;5:e60-e64. http://dx.doi.org/10.5489/ cuaj.10094
12. Robbins SL, Cotran RS, Kumar V, et al. Pathologic Basis of Disease, 6th ed. Philadelphia: WB Saunders Company; 1999:144.

13. Ota T, Kawai K, Hattori K, et al. Hamartoma of the Urinary Bladder. Int I Urol 1999;6:21 1-4. http:// dx.doi.org/10.1046/i.1442-2042.1999.06434.x

14. Hyams ES, Provet J. Angiomyolipoma of the left ureterovesical junction. Rev Urol 2007;9:84-8.

15. Huan Y, Dillon RW, Unger PD. Angiomyolipoma of the bladder. Ann Diagn Pathol 2002;6:378-80. http:// dx.doi.org/10.1053/adpa.2002.36655

16. Wein AJ, Kavoussi LR, Novick AC, et al. Campbell-Walsh Urology, 10th ed. Elsevier: Saunders;2012:2985-9.

17. Shapiro E. Clinical implications of genitourinary embryology. Curr Opin Urol 2009;19:427-33. http:// dx.doi.org/10.1097/MOU.0b013e32832c90ff

Correspondence: Dr. Michael Chua, St. Luke's Medical Center, Institute of Urology, 279 E. Rodriguez Sr. Boulevard, Quezon City, 1112 Philippines; auhc_ekim@yahoo.com 